\title{
No Tissue Expression of KRAS or BRAF Mutations in 61 Adult Patients Treated for Esophageal Atresia in Early Childhood
}

\section{Dang, Kien Xuan}

2018-10

Dang , KX, Ho , T , Sistonen , S , Koivusalo , A, Pakarinen , M , Rintala , R , Stenman , U-H , Orpana , A \& Stenman , J 2018 , ' No Tissue Expression of KRAS or BRAF Mutations in 61 Adult Patients Treated for Esophageal Atresia in Early Childhood ' , European Journal of Pediatric Surgery , vol. 28 , no. 5 , pp. 413-419 . https://doi.org/10.1055/s-0037-1605346

http://hdl.handle.net/10138/311469

https://doi.org/10.1055/s-0037-1605346

unspecified

acceptedVersion

Downloaded from Helda, University of Helsinki institutional repository.

This is an electronic reprint of the original article.

This reprint may differ from the original in pagination and typographic detail.

Please cite the original version. 


\title{
No tissue expression of KRAS or BRAF mutations in biopsies from 61 adult patients treated for Esophageal Atresia in early childhood.
}

Kien X. Dang ${ }^{1, *}$, Tho H. Ho ${ }^{2}$, Saara Sistonen ${ }^{3}$, Antti Koivusalo ${ }^{3}$, Mikko Pakarinen ${ }^{3}$, Risto Rintala ${ }^{3}$, Ulf-Hakan Stenman ${ }^{4}$, Arto Orpana ${ }^{5}$ and Jakob Stenman ${ }^{1,6,7^{*}}$

${ }^{1}$ Minerva Foundation Institute for Medical Research, Helsinki, Fl-00290, Finland; ${ }^{2}$ Genomics Laboratory, Department of genomics and cytogenetics, Vietnam Military Medical University, Hanoi, Vietnam; ${ }^{3}$ Department of Pediatric Surgery, Children's Hospital, Helsinki University Central Hospital, FI-00029, Finland; ${ }^{4}$ Haartman Institute, Department of Clinical Chemistry, Biomedicum Helsinki, University of Helsinki and Helsinki University Central Hospital, Helsinki, FI-00029 HUS, Finland; ${ }^{5}$ Laboratory of Genetics, HUSLAB, Helsinki University Central Hospital, HUSLAB, 00029 Helsinki, Finland. ${ }^{6}$ Institute for Molecular Medicine Finland, University of Helsinki, Helsinki, FI-00014, Finland and ${ }^{7}$ Department of Women's and Children's Health, Karolinska Institute, Stockholm, SE-17176, Sweden

"To whom correspondence should be addressed. Tel: +358 41493 7225; Fax: +358 9191 25701; Email: kien.dang@helsinki.fi. Correspondence may also be addressed to Jakob Stenman, Tel: +46 72 9444028; Fax: +358 9191 25701; Email: jakob.stenman@karolinska .se

\begin{abstract}
Background

Previous studies have reported an association between esophageal atresia, Barrett's esophagus and esophageal adenocarcinoma later in life. In order to look for signs of malignant transformation, we used an ultra-sensitive technique to detect expressed KRAS and BRAF mutations in endoscopic biopsies from 61 adult patients under follow-up after treatment for esophageal atresia.

\section{Methods}

RNA was extracted from 112 fresh frozen endoscopic tissue biopsies from 61 adult patients treated for esophageal atresia in early childhood. RNA was reverse transcribed using the Extendable Blocking Probe Reverse Transcription method. KRAS codon 12 and 13, as well as BRAF mutations were detected by qPCR.

Results

No mutations of KRAS codon 12, KRAS codon 13 or BRAF was found in 112 endoscopic biopsy samples from 61 patients.

\section{Conclusions}

Despite the precence of histological findings indicating long standing gastroesophageal reflux in $25 \%$, as well as symptomatic gastroesophageal reflux in more than $40 \%$, there were no detectable tissueexpression of KRAS or BRAF mutations to indicate malignant transformation in this cohort of patients.
\end{abstract}


Key words: KRAS, BRAF, esophageal atresia, extendable blocking probe.

\section{INTRODUCTION}

Esophageal atresia (EA) occurs with frequency of 1 in 2,500 to 4,500 live births [1-4]. Adult patients born with esophageal atresia have high incidence rates of gastro-esophageal reflux symptoms as well as histological signs of esophagitis and Barrett's esophagus (BE) [5-7]. 2010. BE is known to be a preneoplastic condition and a risk factor for developing esophageal adenocarcinoma (EAC), a very often deadly cancer [8-10]. To date there has been at least five reported cases of EAC in patients treated for esophageal atresia and several investigators have suggested a greatly increased risk of developing EAC in these patients [11].

The RAS-RAF-MEK-ERK-MAP kinase pathway (KRAS-MAPK pathway) is currently regarded as one of the most important pathways in cancer development. The KRAS and BRAF genes are members of the KRASMAPK pathway, and mutations in these genes are found in $30 \%$ and $8 \%$, respectively, of human tumours [12-14]. KRAS and BRAF mutation have been demonstrated to be genetic markers in lung cancer, pancreatic cancer, colorectal cancer, ovarian cancer, including prostate and gastric cancers [15]. The role of these oncogenes in the development and progression of BE into EAC remains unclear [16].

KRAS mutations have been shown to occur in $21-36 \%$ of EAC and in $27-40 \%$ of high grade intraepithelial neoplasia [16-18], whereas BRAF mutations have been reported in $4-11 \%$ of cases with intestinal metaplasia or Barrets esophagus $[16,23,24]$. Most of the reported mutations have been detected in codon 12 of the KRAS gene $[16,18]$. To the best of our knowledge, only 3 cases of KRAS codon 13 mutations have been described in EAC [19-21]. In a large study examining 412 biopsies from patients with Barrett's oesophagus, no KRAS codon 13 mutations were detected [18]. Similarly, only 2 cases of BRAF V600E mutation have been reported in esophageal cancer [22], and one study did not find any BRAF-V600E mutation in 534 gastroesophageal tumors [23]. Although the results from previous studies are slightly divergent, KRAS codon 12 mutations seem to be common in patients with Barrett's esophagus with high-grade dysplasia or EAC but rare or absent in patients with low grade lesions. This suggests that particularly KRAS codon 12 mutations might be useful as a genetic marker for screening patients at risk of adenocarcinoma transformation [24] Currently there are no studies on KRAS and BRAF mutations in patients treated for EA.

Previous studies examining KRAS and BRAF mutations in BE and EAC have utilized DNA-based detection techniques, such as sequencing or selective polymerase chain reaction $[16,17]$. We have recently described a novel method for ultra-sensitive detection of expressed mutations by extendable blocking probe reverse transcription (ExBP-RT) This technique can detect single nucleotide polymorphisms and point mutation with a high sensitivity and selectivity in comparison to other methods [25]. The ExBP-RT technique uses RNA as starting material instead of DNA, thus reflecting tissue expression of mutated genes rather than the presence of mutated DNA in the sample. The relatively high abundance of RNA in tissue samples, as well as the high sensitivity of this technique enables ultra-sensitive detection of expressed mutations, which allows detection of mutations in a minimal subset of cells in a tissue 
sample. In order to determine the incidence and evaluate KRAS and BRAF mutations as genetic markers for malignant transformation in patients previously treated for esophageal atresia, we have utilized the ExBP-RT method to identify tissue expression of KRAS and BRAF mutations in endoscopic biopsies from adult patients treated for EA in early childhood.

\section{MATERIAL AND METHODS}

\section{Patients and clinical data}

During the time period 2001-2004, endoscopic biopsies were retrieved from 61 patients treated for esophageal atresia in early childhood at the Hospital for Children and Adolescents, Helsinki University Central Hospital. RNA was successfully isolated from biopsy specimen taken from 61 of these patients. The age at biopsy was 21-56 years (mean 35,6, median 32,7 years). 35 of the patients were male and 26 were female. The distribution of the type of esophageal atresia was; Esophageal atresia with proximal tracheo-esophageal fistula (TEF): 1,6\%, distal TEF: 90,2\%, proximal and distal TEF 4,9\%, H-type TEF 3,3\%. Six out of 61 patients had a long-gap disease as defined by a distance between the upper and lower pouch of more than $3 \mathrm{~cm}$ or at least two vertebral bodies on plain radiography. 10/61 patients had tracheomalacia. Six patients had been re-operated due to a fistula. 59/61 patients in this cohort had undergone dilatations of the esophageal anastomosis (range $0-66$, mean 11,8 , median 8 ). Four patients had undergone a surgical resection of a stricture and 10 patients had undergone a fundoplication. Histological examination of the biopsy specimens showed esophagitis in 5/61 cases, gastric metaplasia in 10/61 cases and intestinal metaplasia in 5/61 cases. Informed consent was received from all patients participating in the study in accordance with the Helsinki Declaration. The study was approved by the Ethics Committee of the Hospital for Children and Adolescents, University of Helsinki, Finland.

\section{RNA samples}

RNA was extracted from 112 fresh frozen endoscopic tissue biopsies from 61 patients using phenolchloroform extraction [26]. All RNA samples were quantified with a NanoVue spectrophotometer (GE Healthcare, Waskesha, WI) and diluted to $100 \mathrm{ng} / \mu \mathrm{l}$ in Diethylpyrocarbonate (DEPC) $\mathrm{H}_{2} \mathrm{O}$, before the extendable blocking probe reverse transcription (ExBP-RT) reaction.

RNA extracted from A549, Lovo and Colo205 cell lines were used as positive controls. Table 1. All control RNAs were extracted from cell cultures using RNA/DNA purification Kit (Norgen Biotek) then quantified with a NanoVue spectrophotometer and diluted to $100 \mathrm{ng} / \mu \mathrm{l}$ in DEPC H2O before using.

Table 1. Cell line RNA used as positive controls

\begin{tabular}{|l|l|l|l|l|l|}
\hline Cell line & Mutation & $\begin{array}{l}\text { KRAS Codon 12 } \\
\text { mutation analysis }\end{array}$ & $\begin{array}{l}\text { KRAS Codon 13 } \\
\text { mutation analysis }\end{array}$ & $\begin{array}{l}\text { BRAF mutation } \\
\text { analysis }\end{array}$ & $\begin{array}{l}\text { Concen- } \\
\text { tration }\end{array}$ \\
\hline A549 & KRAS - G12R & Mutant control & - & $\begin{array}{l}\text { Wild-type } \\
\text { control }\end{array}$ & $100 \mathrm{ng} / \mu \mathrm{l}$ \\
\hline Lovo & KRAS - G13D & - & Mutant control & - & $100 \mathrm{ng} / \mu \mathrm{l}$ \\
\hline
\end{tabular}




\begin{tabular}{|l|l|l|l|l|l|}
\hline Colo205 & BRAF - V600E & Wild-type control & Wild-type control & Mutant control & $100 \mathrm{ng} / \mu \mathrm{l}$ \\
\hline
\end{tabular}

\section{ExBP-RT based mutation detection assays:}

Extendable Blocking Probe Reverse Transcription (ExBP-RT)[25] was employed for ultrasensitive detection of most common mutations of the KRAS and BRAF genes. Using RNA templates, this novel method allows for detection of expressed mutations in at least 1000 times more excessive background of the corresponding wild type alleles. Mutations covered in this study include six different common KRAS mutations at codon 12 (G12D, G12A, G12V, G12S, G12A, G12C); one KRAS mutation at codon 13 (G13D); and one BRAF mutation (V600E). The principles of ExBP-RT assays and reaction set-up procedures for multiplex detection of six KRAS mutations at codon 12 and one BRAF mutation (V600E) were as described in the original paper [25]. ExBP-RT primers and probe for detection of KRAS mutation at codon 13 (G13D) are listed in Table 3.

Table 3. Primer, blocking probe for ExBP-RT assays of mutant KRAS codon 13 (Locked Nucleic Acid (LNA) $=[+A],[+G],[+C],[+T])$.

\begin{tabular}{|l|l|l|}
\hline Primers and probes & $\begin{array}{l}\text { Sequences } \\
\text { (The engineering 5'-tail sequences in bold) }\end{array}$ & Concentrations \\
\hline \multicolumn{2}{|l|}{ KRAS codon 13 Mutation-specific primer } & $0.5 \mu \mathrm{M}$ \\
\hline KRAS G13D (GGC>GAC) & 5'-CGATCAGACGACGACTATTATTACG[+T]CACC-3' & \multicolumn{2}{|l}{} \\
\hline KRAS codon 13 Wildtype-specific blocking probe & $4 \mu \mathrm{M}$ \\
\hline KRAS13-INERT & 5'-CTACGCCACCA-3' &
\end{tabular}

\section{Detection and quantification of expressed mutations}

Using products of ExBP-RT assays as template, real-time PCR amplification were performed to detect/quantify expressed mutations of KRAS and BRAF genes. QuantiTect Probe PCR Kits (QIAGEN) were used for these probe-based real-time PCR assays according to the manufacturer's instructions in a $10 \mu \mathrm{L}$ volume of reaction. The Taqman probes for simultaneous detection of six different KRAS mutations at codon 12 were designed to contain 2 universally binding inosine nucleotides that allows for targeting the variants nucleotides of different types of mutation. A common reverse primer was designed to target the 5'-prime tail of all mutation-specific ExBP-RT products. The expression levels of total KRAS and total BRAF genes (both mutant and wild type) were also determined in each sample for normalization using QuantiTect SYBR ${ }^{\circledast}$ Green PCR Kits (QIAGEN) according to the manufacturer's instructions in a $10 \mu \mathrm{L}$ volume. The sequences and concentration of qPCR primers and probes are 
provided in Table 4. The same thermocycling conditions were used for both probe-based and SYBRbased qPCR: $95^{\circ} \mathrm{C}$ for $15 \mathrm{~min}, 45 \mathrm{cycles}$ at $94^{\circ} \mathrm{C}$ for $10 \mathrm{sec}$, at $60^{\circ} \mathrm{C}$ for $45 \mathrm{sec}$. Following SYBR-based qPCR, the specificity of the amplification products was always verified by melting curve analysis. All qPCR assays were run on a LightCycler 480 II Real-Time PCR Instrument (Roche Diagnostics Oy, Finland) with 384-well white-plate. All mutation controls, wild type controls and $\mathrm{H} 2 \mathrm{O}$ controls of each performed experiment were checked to verify the correction of results in both ExBP-RT, qPCR assays and avoid the problem of contamination.

Table 4. Primer and probe sequences for qPCR step of different ExBP-RT assays (Locked Nucleic Acid $($ LNA $)=[+A],[+G],[+C],[+T]$; Inosine = i; 6-carboxyfluorescein: FAM; Black Hole Quenchers: BHQ).

\begin{tabular}{|c|c|c|}
\hline Primers and probes & Sequences $\left(5^{\prime}-3^{\prime}\right)$ & Concentrations \\
\hline \multicolumn{3}{|c|}{ Mutant KRAS codon 12 assays } \\
\hline KRAS Forward primer & 5'-CCTGCTGAAAATGACTGAA-3' & $0.5 \mu \mathrm{M}$ \\
\hline Common Reverse primer & 5'-CGATCAGACGACGAC-3' & $0.5 \mu \mathrm{M}$ \\
\hline KRAS12-Probe & FAM-AT[+T]A[+T]T[+C]C[+A]ii $[+A] G[+C] T C C-B H Q 1$ & $0.1 \mu \mathrm{M}$ \\
\hline \multicolumn{3}{|l|}{ Mutant KRAS codon 13 assays } \\
\hline KRAS Forward primer & 5'-CCTGCTGAAAATGACTGAA-3' & $0.5 \mu \mathrm{M}$ \\
\hline Common Reverse primer & 5'-CGATCAGACGACGAC-3' & $0.5 \mu \mathrm{M}$ \\
\hline KRAS13-Probe & FAM-AGC $[+T] G G[+T] G A[+C] G[+T] A A[+T] A A T-B H Q 1$ & $0.1 \mu \mathrm{M}$ \\
\hline \multicolumn{3}{|l|}{ Total-KRAS assays } \\
\hline KRAS Forward primer & 5'-CCTGCTGAAAATGACTGAA-3' & $1.5 \mu \mathrm{M}$ \\
\hline Total KRAS Reverse primer & 5'-GCCACCAGCTCCAACTACCACAA-3' & $1.5 \mu \mathrm{M}$ \\
\hline \multicolumn{3}{|l|}{ Mutant BRAF assays } \\
\hline BRAF Forward primer & 5'-AGACCTCACAGTAAAAATAGGTGA-3' & $0.5 \mu \mathrm{M}$ \\
\hline
\end{tabular}




\begin{tabular}{|c|c|c|}
\hline Common Reverse primer & 5'-CGATCAGACGACGAC-3' & $0.5 \mu \mathrm{M}$ \\
\hline BRAF-Probe & FAM-TTC $[+\mathrm{T}] \mathrm{CT}[+\mathrm{G}] \mathrm{TA}[+\mathrm{G}] \mathrm{CT}[+\mathrm{A}] \mathrm{GACCAA}-\mathrm{BHQ} 1$ & $0.1 \mu \mathrm{M}$ \\
\hline \multicolumn{3}{|l|}{ Total-BRAF assays } \\
\hline Total BRAF Forward primer & 5'-CATGAAGACCTCACAGTAAA-3' & $1.5 \mu \mathrm{M}$ \\
\hline Total BRAF Reverse primer & 5'-GATTTCACTGTAGCTAGACC-3' & $1.5 \mu \mathrm{M}$ \\
\hline
\end{tabular}

We calculated threshold cycle $(\mathrm{Ct})$ values of qPCR automatically using the Absolute Quantification Analysis with the Fit Points Method, which is built in the LightCycler ${ }^{\circledR} 480$ II system (Roche Diagnostics Oy, Finland). That allows to set the noiseband and the threshold line in order to discard uninformative background noise.

\section{RESULTS}

In this study, we examined 112 fresh frozen endoscopic tissue biopsies from 61 adult patients under follow-up after surgical treatment for esophageal atresia. In order to verify the viability of the RNA samples, the total tissue expression levels of the KRAS and BRAF genes were analysed by real-time PCR with primer pairs that co-amplifies both mutant and wild type cDNAs generated in ExBP-RT assays. Tissue expression of the KRAS and BRAF genes could be readily detected in all 112 samples (Figure 1). Mutation controls consisting of RNA extracted from cell lines that express KRAS mutation at codon 12 (A549), KRAS mutation at codon 13 (Lovo) and BRAF mutation (Colo 205) could be readily detected in every experiment (Figure 2). Expression of KRAS codon 12, codon 13 or BRAF mutations was, however, not detected in any of the 112 patient samples. 

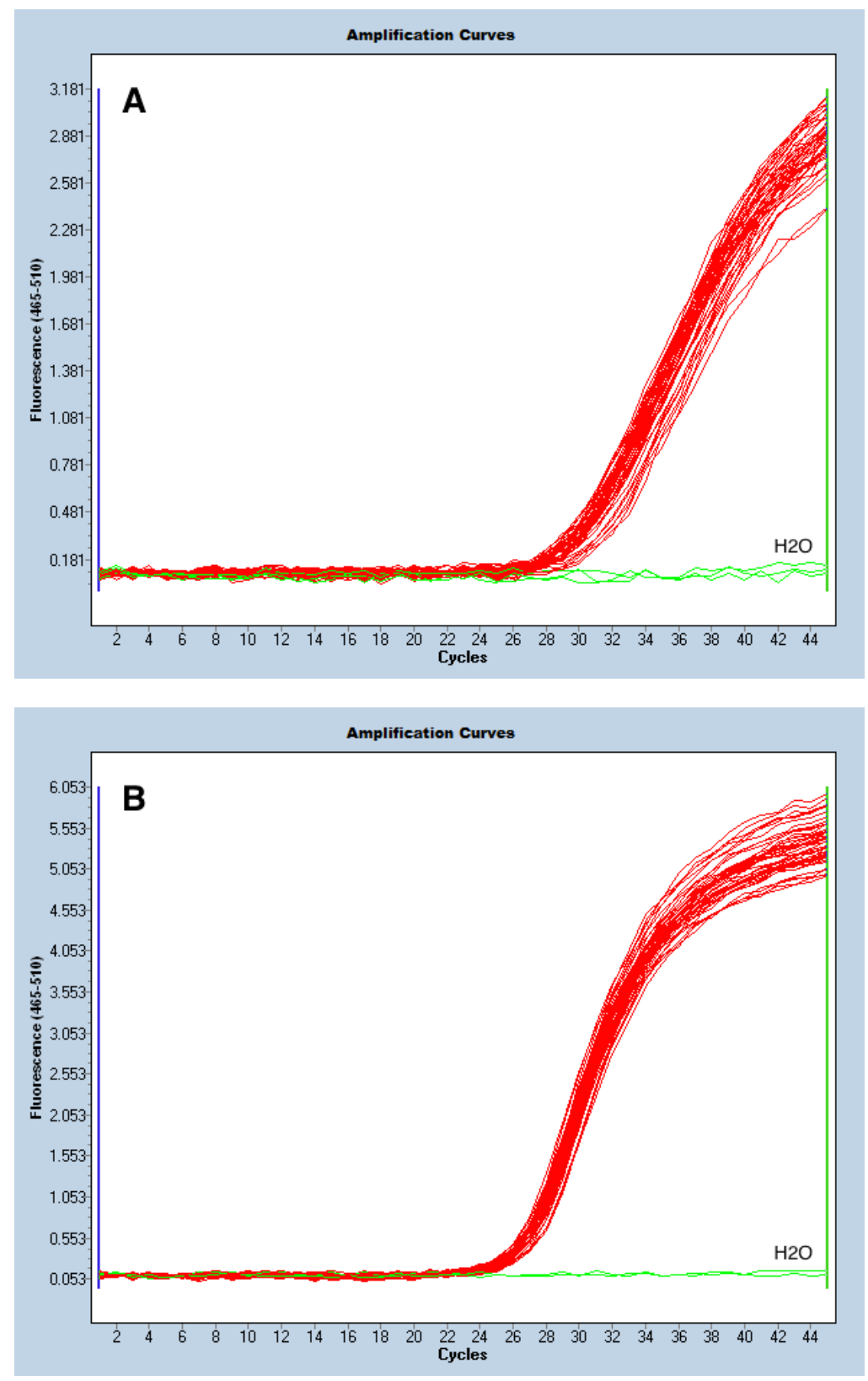

Figure 1. (A) Total KRAS detection in qPCR. (B) Total BRAF detection in qPCR. 

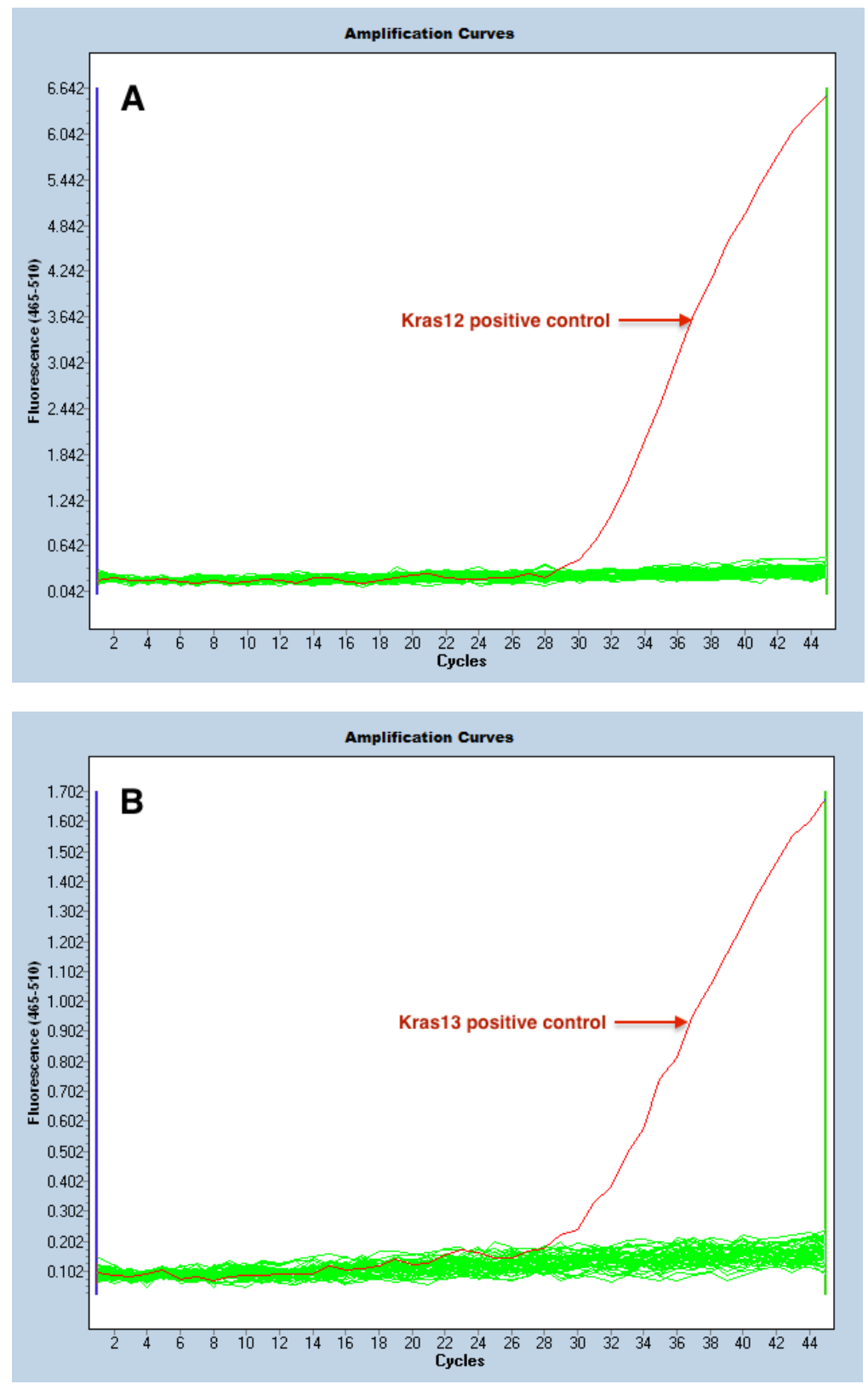


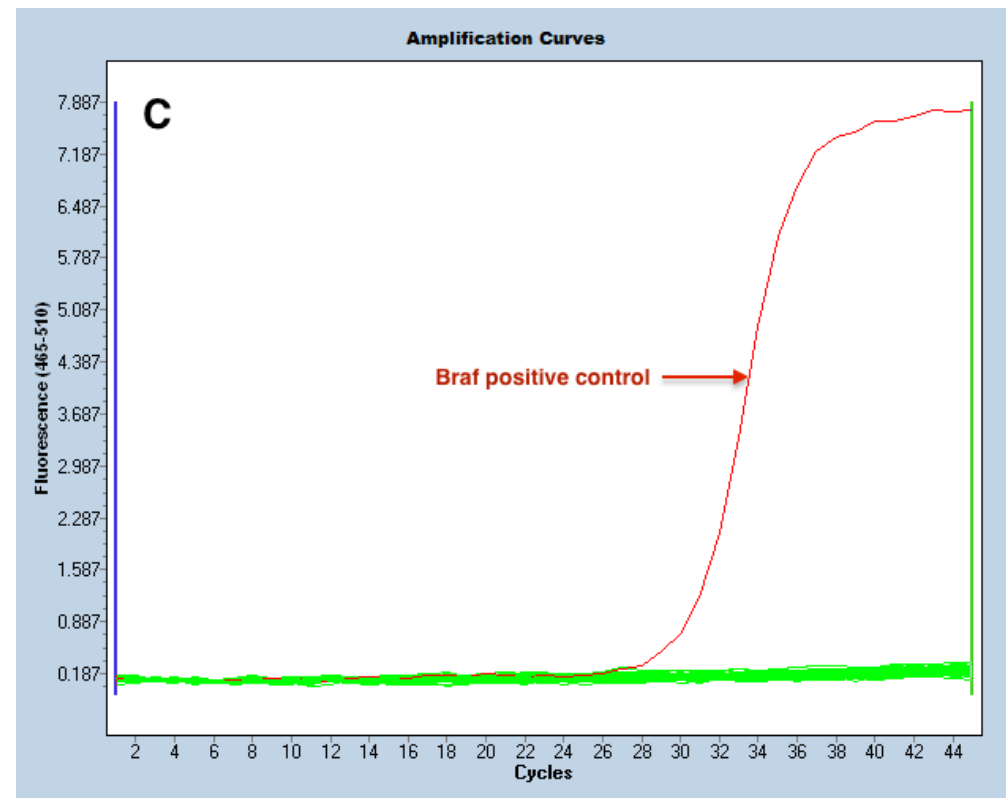

Figure 2. (A) KRAS codon 12 mutation detection in qPCR. (B) KRAS codon 13 mutation detection in qPCR. (C) BRAF mutation detection in $\mathrm{QPCR}$. 


\section{DISCUSSION}

EA is a developmental disorder of the esophagus, where in most cases, a proportion of the esophagus is missing. The innervation of the distal part of the esophagus is inherently interrupted and surgical repair of EA is not believed to restore innervation. A certain degree of gastro-esophageal reflux disease (GERD) thus, occurs from birth in most EA patients and this is thought to play a role in the development of Barrett's esophagus (BE) through repeated mucosal damage [24, 27]. Replacement of the the normal squamous epithelium by intestinalized columnar epithelium in Barrett's esophagus is considered a preneoplastic condition that predisposes to EAC [8] and the association between EA, BE and EAC has been well established $[5,24]$. In this study we strived to clarify whether KRAS or BRAF mutations would be present at detectable levels in patients previously treated for EA. The rationale was that the presence of expressed KRAS or BRAF mutations, reflecting signs of malignant transformation, could be used for identifying patients at risk of developing EAC. For this purpose, we utilized a novel RNA-based technique, ExBP-RT , for point mutation detection with high sensitivity compared to DNA-based techniques [25]. We have previously shown that KRAS codon 12 mutations could be detected in in 16/44 (36.4\%) archival colorectal cancer FFPE specimens. This corresponds to a slightly higher mutation rate than in previous reports (27.7\%) using DNA-based mutation detection assays [28] and subsequent analyses of 775 samples are coherent with these findings (unpublished results).

KRAS and BRAF mutations are predominantly found in cancers arising from abnormal columnar epithelium, such as colorectal cancer and pancreatic cancer. KRAS mutations are found in $30-60 \%$ of colorectal cancers [29-31] and they are present in more than $90 \%$ of pancreatic ductal adenocarcinomas (PDAC) [32]. BRAF mutations are present in colorectal cancer with frequency of 10-15\% [33]. In esophageal squamous cell carcinomas (ESCCs) and Basaloid squamous cell carcinomas (BSCCs) KRAS and BRAF mutations are rare or absent [34-37], suggesting that these mutations play a limited role in the development of ESCC [34]. In EAC and associated high grade intraepithelial lesions, however, KRAS codon 12 mutations occur at a frequency between 30 and $40 \%$, and might represent a late event in the Barrett's metaplasia-dysplasia-adenocarcinoma sequence [16]. KRAS codon 13 and BRAF mutations are known to play a less important role in the development of digestive tract cancers. These mutations seem to be rare in EAC and may not be involved in the development of Barrett's esophagus and EAC. We have established ExBP-RT assays for detection of the six most common mutations at KRAS codons 12 and 13, and one common mutation of BRAF V600. These mutations represent $97 \%$ (94.5-99.6\%) of KRAS and $72 \%(47-96 \%)$ of BRAF mutations that have been described. To the best of our knowledge, this is the first study describing the mutational status of KRAS and BRAF in patients treated for EA.

In this study we could not detect tissue expression of KRAS or BRAF mutations in any of the 112 endoscopic biopsies from 61 patients treated for EA. While histological analysis revealed gastric metaplasia in 10/61 cases and intestinal metaplasia in further 5/61 cases, neither dysplasia nor EAC was found in any of these patients. In a previous study by Trautmann et al., KRAS codon 12 mutations were only found in 1/252 (0.4\%) metaplasia patients and 4/105 (3.8\%) dysplasia patients [18]. In addition, Aber et al. detected KRAS mutations in only $1 / 39$ (2\%) patients with Barrett's esophagus. [35] Our results are, consistent with these findings, implying that KRAS codon 12 mutations are rare or absent in early stages of esophageal adenocarcinoma. [16] It is possible that the KRAS and BRAF genes might not 
be ideal markers for malignant transformation in patients with EA, and that an assay for P53-mutations, which are the most common mutations found in EAC might serve as a more reliable genetic marker. On the other hand, the negative result reported here might also reflect the fact that no malignant transformation had taken place in any of the 61 patients included in the study. The aim of this study was to identify genetic markers that would identify patients in need of close follow-up or early intervention. At the time of sampling, the mean age of this cohort of patients was 35,6 years. Future studies with a longer follow-up time might be helpful to shed light on these questions.

In conclusion, we have utilized a highly sensitive technique to detect tissue expression on mutated KRAS and BRAF genes in endoscopic esophageal biopsies from patients previously treated for EA. The absence of KRAS, BRAF mutations gives no indication of malignant transformation in this cohort of patients. We hope that this study can serve as an inspiration for developing techniques for preventive follow-up of patients treated for esophageal atresia.

\section{ACKNOWLEDGMENT}

We thank Anne Ahmanheimo for excellent technical assistance.

\section{FUNDING}

Centre for International Mobility (CIMO) fellowships; Finska Läkare Sällskapet; and Minerva Foundation for Medical Research. Funding for open access charge: University of Helsinki.

\section{CONFLICT OF INTEREST}

None. 


\section{REFERENCES}

1. $\quad$ Spitz, L., Oesophageal atresia. Orphanet J Rare Dis, 2007. 2: p. 24.

2. Depaepe, A., H. Dolk, and M.F. Lechat, The epidemiology of tracheo-oesophageal fistula and oesophageal atresia in Europe. EUROCAT Working Group. Arch Dis Child, 1993. 68(6): p. 743-8.

3. Torfs, C.P., C.J. Curry, and T.F. Bateson, Population-based study of tracheoesophageal fistula and esophageal atresia. Teratology, 1995. 52(4): p. 220-32.

4. Holland, A.J. and D.A. Fitzgerald, Oesophageal atresia and tracheo-oesophageal fistula: current management strategies and complications. Paediatr Respir Rev, 2010. 11(2): p. 100-6; quiz 1067.

5. Krug, E., et al., Gastroesophageal reflux and Barrett's esophagus in adults born with esophageal atresia. Am J Gastroenterol, 1999. 94(10): p. 2825-8.

6. Koivusalo, A., M.P. Pakarinen, and R.J. Rintala, The cumulative incidence of significant gastrooesophageal reflux in patients with oesophageal atresia with a distal fistula--a systematic clinical, pH-metric, and endoscopic follow-up study. J Pediatr Surg, 2007. 42(2): p. 370-4.

7. Sistonen, S.J., et al., Esophageal morbidity and function in adults with repaired esophageal atresia with tracheoesophageal fistula: a population-based long-term follow-up. Ann Surg, 2010. 251(6): p. 1167-73.

8. Maltby, E.L., et al., Molecular abnormalities in pediatric barrett esophagus: can we test for potential of neoplastic progression? Pediatr Dev Pathol, 2010. 13(4): p. 310-7.

9. Shaheen, N.J. and J.E. Richter, Barrett's oesophagus. Lancet, 2009. 373(9666): p. 850-61.

10. Koppert, L.B., et al., The molecular biology of esophageal adenocarcinoma. J Surg Oncol, 2005. 92(3): p. 169-90.

11. Sistonen, S.J., et al., Cancer after repair of esophageal atresia: population-based long-term follow-up. J Pediatr Surg, 2008. 43(4): p. 602-5.

12. Davies, H., et al., Mutations of the BRAF gene in human cancer. Nature, 2002. 417(6892): p. 94954.

13. Cantwell-Dorris, E.R., J.J. O'Leary, and O.M. Sheils, BRAFV600E: implications for carcinogenesis and molecular therapy. Mol Cancer Ther, 2011. 10(3): p. 385-94.

14. Samatar, A.A. and P.I. Poulikakos, Targeting RAS-ERK signalling in cancer: promises and challenges. Nat Rev Drug Discov, 2014. 13(12): p. 928-42.

15. Vakiani, E. and D.B. Solit, KRAS and BRAF: drug targets and predictive biomarkers. J Pathol, 2011. 223(2): p. 219-29.

16. Lord, R.V., et al., K-ras codon 12 mutations in Barrett's oesophagus and adenocarcinomas of the oesophagus and oesophagogastric junction. J Gastroenterol Hepatol, 2000. 15(7): p. 730-6.

17. Sommerer, F., et al., Mutations of BRAF and KRAS2 in the development of Barrett's adenocarcinoma. Oncogene, 2004. 23(2): p. 554-8.

18. Trautmann, B., et al., $K$-ras point mutations are rare events in premalignant forms of Barrett's oesophagus. Eur J Gastroenterol Hepatol, 1996. 8(8): p. 799-804.

19. Janmaat, M.L., et al., Predictive factors for outcome in a phase I/ study of gefitinib in second-line treatment of advanced esophageal cancer patients. J Clin Oncol, 2006. 24(10): p. 1612-9.

20. Okines, A.F., et al., Biomarker analysis in oesophagogastric cancer: Results from the REAL3 and TransMAGIC trials. Eur J Cancer, 2013. 49(9): p. 2116-25.

21. Chong, I.Y., et al., The genomic landscape of oesophagogastric junctional adenocarcinoma. J Pathol, 2013. 231(3): p. 301-10.

22. Sekine, S., et al., Esophageal melanomas harbor frequent NRAS mutations unlike melanomas of other mucosal sites. Virchows Arch, 2009. 454(5): p. 513-7. 
23. Preusser, M., et al., No evidence for BRAF-V600E mutations in gastroeosophageal tumors: results from a high-throughput analysis of 534 cases using a mutation-specific antibody. Appl Immunohistochem Mol Morphol, 2013. 21(5): p. 426-30.

24. Schneider, A., L. Michaud, and F. Gottrand, Esophageal atresia: metaplasia, Barrett. Dis Esophagus, 2013. 26(4): p. 425-7.

25. Ho, T.H., et al., Extendable blocking probe in reverse transcription for analysis of RNA variants with superior selectivity. Nucleic Acids Res, 2015. 43(1): p. e4.

26. Stenman, J., et al., Targeted gene-expression analysis by genome-controlled reverse transcription-PCR. Clin Chem, 2006. 52(11): p. 1988-96.

27. Gottrand, F., et al., [Outcome of children with repaired oesophageal atresia]. Arch Pediatr, 2008. 15(12): p. 1837-42.

28. Andreyev, H.J., et al., Kirsten ras mutations in patients with colorectal cancer: the multicenter "RASCAL" study. J Natl Cancer Inst, 1998. 90(9): p. 675-84.

29. Bos, J.L., ras oncogenes in human cancer: a review. Cancer Res, 1989. 49(17): p. 4682-9.

30. Kressner, U., et al., Ki-ras mutations and prognosis in colorectal cancer. Eur J Cancer, 1998. 34(4): p. 518-21.

31. Fearon, E.R. and B. Vogelstein, A genetic model for colorectal tumorigenesis. Cell, 1990. 61(5): $\mathrm{p}$. 759-67.

32. Eser, S., et al., Oncogenic KRAS signalling in pancreatic cancer. Br J Cancer, 2014. 111(5): p. 81722.

33. Chen, J., et al., BRAF V600E mutation and KRAS codon 13 mutations predict poor survival in Chinese colorectal cancer patients. BMC Cancer, 2014. 14: p. 802.

34. Shigaki, H., et al., KRAS and BRAF mutations in 203 esophageal squamous cell carcinomas: pyrosequencing technology and literature review. Ann Surg Oncol, 2013. 20 Suppl 3: p. S485-91.

35. Arber, N., et al., Activation of $c-K$-ras mutations in human gastrointestinal tumors. Gastroenterology, 2000. 118(6): p. 1045-50.

36. Baba, Y., et al., Molecular Characteristics of Basaloid Squamous Cell Carcinoma of the Esophagus: Analysis of KRAS, BRAF, and PIK3CA Mutations and LINE-1 Methylation. Ann Surg Oncol, 2015.

37. Maeng, C.H., et al., High-throughput genotyping in metastatic esophageal squamous cell carcinoma identifies phosphoinositide-3-kinase and BRAF mutations. PLoS One, 2012. 7(8): p. e41655. 